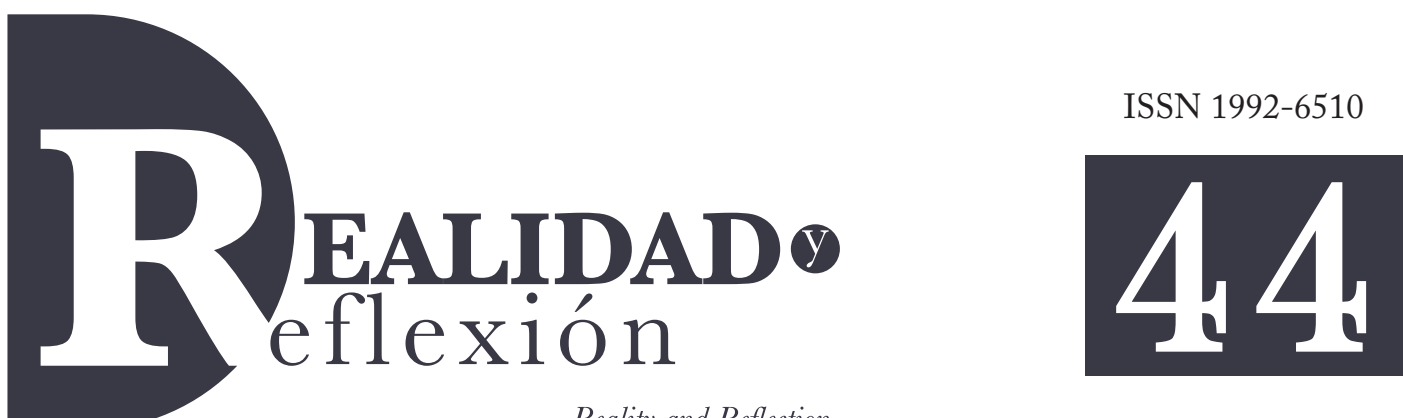

Reality and Reflection

Año 16, N 44, San Salvador, El Salvador, Centroamérica. Revista Semestral Julio-Diciembre 2016

YEAR 16, N 44, SAN SALVADOR, EL SALVADOR, CENTRAL AMERICA. SEMESTRAL JOURNAL JULY-DECEMBER 2016

\title{
Estudio de materiales grafíticos comunes como aceleradores de evaporación de agua bajo irradiación luminosa
}

\author{
Study of commons graphitic materials as enhancers for \\ water evaporation under light irradiation
}

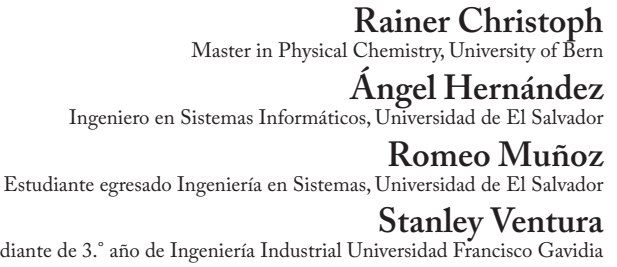

Todos los autores trabajan en el Laboratorio de Nanotecnología de la Universidad Francisco Gavidia

\section{RESUMEN}

Recientemente se ha descubierto que la tasa de evaporación de agua puede aumentar considerablemente, hasta por un factor de 3, utilizando un material con amplio espectro de absorción de luz visible como el grafeno. En el Laboratorio de Nanotecnología de la Universidad Francisco Gavidia de El Salvador se han realizado pruebas con diferentes tipos de grafito disponibles localmente para determinar cuál de ellos es el más eficiente para la evaporación del agua. Este estudio tiene como objetivo utilizar este método para la desalinización de agua de mar utilizando menos energía y aumentando la velocidad de obtención de agua potable. Para los experimentos se ha utilizado tecnología de impresión 3D como desarrollo de prototipado rápido.

Palabras clave: desalinización, grafito, impresión 3D.

ABSTRACT

Recently it has been discovered that the rate of water evaporation can increase significantly up to a factor 3 using a material with broad absorption spectrum of visible light such as grapheme. At the Nanotechnology Laboratory of Francisco Gavidia University, El Salvador. We have tested with different types of graphite available locally to determinate which of them is more efficient to evaporate water. The goal of this study is desalination of seawater using less energy and increasing the speed of obtaining drinking water. We have used $3 D$ printing technology as development of rapid prototyping.

Keywords: graphitic materials, desalination of seawater, 3D printing. 


\section{Introducción}

La capacidad de desalinizar aguas marinas para generar agua potable representa una solución tecnológica de relevancia vital para El Salvador, cuyas aguas territoriales abarcan un múltiple de su extensión de tierra firme (El Salvador Mi Pais, s.f.) y donde muchas fuentes de agua ubicadas en las costas se ven afectadas por altos grados de contaminación (Cuéllar, 2001). Por lo tanto, es importante familiarizar a presentes y futuras generaciones de profesionales en el país, con las diferentes metodologías de desalinización existentes; pero también mantenerlos informados acerca de nuevos desarrollos en esta temática, especialmente aquellos impulsados por el aporte de las nanotecnologías, que contribuyen a una mayor eficiencia energética y reducciones del impacto ambiental y los costos.

Uno de los métodos de desalinización de aguas marinas más antiguos utilizados por el hombre (Aristóteles, 350 a.C.) y la naturaleza consiste en su destilación, usando el Sol como fuente de energía. La eficiencia de este proceso depende fuertemente de la tasa de evaporación de agua que se logre obtener por unidad de área de superficie $\left(\mathrm{m}^{2}\right)$ expuesta a la fuente de radiación. Entre más cantidad de agua se logra evaporar por $\mathrm{m}^{2}$ de área de superficie expuesta, más alto es el rendimiento de la producción de agua desalinizada.

Desalinizadores solares convencionales usan volúmenes de agua cuyas superficies son expuestas a la radiación solar directa. En este método, la irradiación calienta el agua incrementando la velocidad promedio de translación de sus moléculas individuales y aumentando el número de las mismas, con la suficiente energía cinética para traspasar la superficie expuesta al aire y transitarla a la fase gaseosa. Una de las desventajas de este método tradicional es su alto consumo de energía, ya que gran parte del agua expuesta a la irradiación es calentada sin lograr su evaporación (ver Figura n.1).

En un descubrimiento reciente, científicos del grupo de investigadores del Departamento de Ingeniería Mecánica del MIT, EE.UU. (Ghasemi, 2014), han demostrado que la tasa de evaporación de agua puede aumentar considerablemente hasta por un factor de 3 , mediante la presencia de un material bidimensional con amplio espectro de absorción de luz visible como el grafeno (Ruquan Ye, 2015) en la superficie expuesta a la irradiación.

Para la explicación de este efecto hoy se postula la creación de sitios calientes ("hot spots") en el material absorbente, con temperaturas mucho más altas que el punto de ebullición del agua, mediante la generación de plasmones (Neumann O, 2013) (Han D., 2011). La alimentación continua de estas zonas con agua líquida es explicada por la presencia de canales de dimensiones nanométricas ("nanocanales" (Cohen-Tanugi D. 2012)(E.W. Webster, s.f)) que se forman entre las diferentes partículas de grafeno, así como la combinación de propiedades hidrofóbicas e hidrofílicas de los materiales utilizados (Ghasemi, 2014).

\section{Metodología}

En este estudio se han realizado mediciones cuantitativas de la tasa de evaporación de agua expuesta a radiación luminosa, tanto solar como artificial, utilizando superficies de agua inalteradas y superficies recubiertas con películas de grafito, proveniente de diferentes fuentes de grafito disponibles localmente (ver Tabla 1 ). 


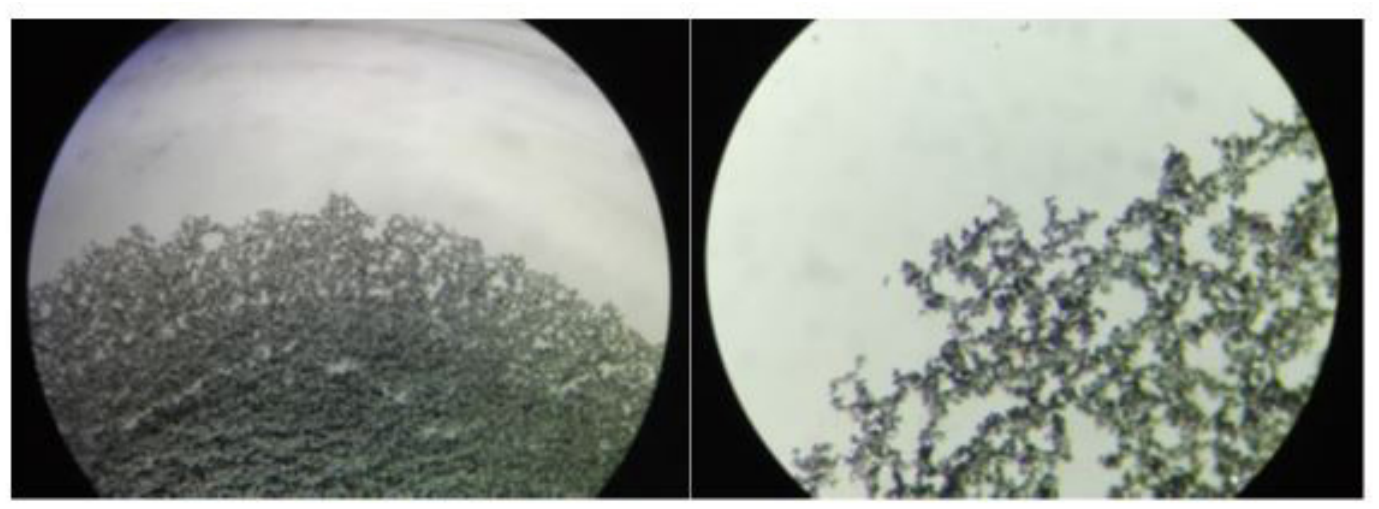

Figura n. $^{\circ}$ 1. Película de polvo negro de grafito de batería flotando sobre la superficie del agua. Este grafito fue tratado en el microondas durante siete segundos antes de ser colocado en el agua. La imagen izquierda muestra un área ampliada 20x mientras que la derecha presenta una ampliación a 100x. Se observa una fuerte cohesión entre las partículas individuales, dando lugar a superficies de agua recubiertas como no recubiertas de grafito, como está ilustrado en la Figura n. ${ }^{\circ} 5$.

\section{Tabla 1}

Descripción de los diferentes materiales grafíticos utilizados

\begin{tabular}{|c|c|c|c|c|}
\hline Material A & Material B & Material C & Material D & Material E \\
\hline $\begin{array}{c}\text { Electrodo } \\
\text { de grafito }\end{array}$ & $\begin{array}{c}\text { Electrodo de } \\
\text { batería } \\
\text { (Rayovac 1.5V) }\end{array}$ & Carbón activado & $\begin{array}{c}\text { Grafito de lápiz } \\
(\text { HB, Facela })\end{array}$ & $\begin{array}{c}\text { Grafito en polvo } \\
\text { (lubricante de } \\
\text { cerraduras) }\end{array}$ \\
\hline
\end{tabular}

Las muestras de los materiales en polvo fueron coladas (apertura de malla: $40 \mu \mathrm{m}$ ) y expuestas durante 7 segundos a la radiación de un microondas, como medida para evacuar gases atrapados en el interior de las partículas y aumentar su superficie activa (Ghasemi, 2014). Las películas de grafito flotante fueron generadas en una superficie circular de agua de $0.0015 \mathrm{~m}^{2}$ con una profundidad de $0.0032 \mathrm{~m}$, colocando 5 gramos de agua (agua desmineralizada ${ }^{1}$ ) para baterías

\footnotetext{
1 Agua desmineralizada, comprada en estación de servicio Puma, San Salvador, El Salvador.
}

con $4 \%$ peso en $\mathrm{NaCl}^{2}$ contenida en una probeta cilíndrica (diámetro: $42 \mathrm{~mm}$ ) y añadiendo el polvo de la muestra en la superficie de agua mediante un dosificador. La distribución homogénea del material flotante fue realizada mediante acción mecánica, hasta formar un recubrimiento completo. El exceso de material en polvo sobre la superficie fue removido mediante un flujo de aire.

La tasa de evaporación se determinó con el arreglo experimental representado en la Figura

\footnotetext{
2 Sal de cocina, comprada en un supermercado local.
} 

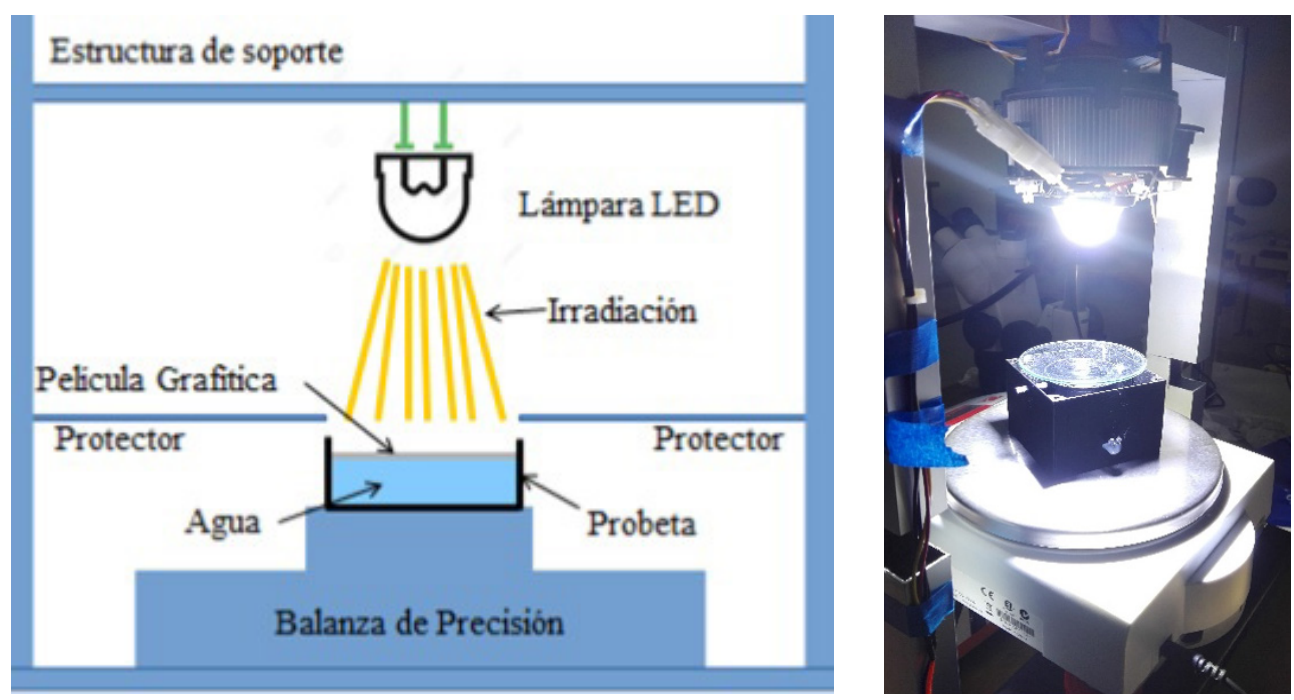

Figura.$^{\circ}{ }^{2}$. Sistema de medición utilizado para determinar las tasas de evaporación de agua bajo irradiación. Derecha: Esquemática del arreglo experimental. Izquierda: Imagen del sistema de medición de evaporación utilizado para esta investigación.

n. 2 , diseñado para mediciones del cambio de peso en tiempo, de probetas con un contenido inicial de 5.00 gramos de agua. Como fuentes de luz se utilizaron una lámpara LED de 100 Vatios (SILED, s.f.), ubicada a una distancia de $120 \mathrm{~mm}$ de la superficie de la película flotante contenida en la probeta, así como también luz solar directa. La pérdida de peso del contenido de agua de las probetas fue medida mediante una balanza electrónica, con una precisión de 0.01 gramos (TAMEX. s.f). La placa base de la balanza electrónica fue aislada de la radiación para evitar recalentamiento y minimizar corrientes de aire provenientes del ventilador de la lámpara LED, evitando posibles repercusiones en los resultados de medición (ver Figura n. ${ }^{\circ}$ ).

Las lecturas de cambio de peso, bajo exposición a la luz fueron realizadas cada 5 minutos, durante el lapso total de una hora. Durante algunos ensayos el transiente de la temperatura del agua contenida en las probetas fue medido mediante un termocupla con salida digital.

Todos los ensayos fueron realizados a una temperatura de ambiente de $25^{\circ} \mathrm{C}$ (temperatura de regulación del aire acondicionado del laboratorio) y con una humedad de aire de aproximadamente 90\%, dictada por las condiciones climatológicas durante la época de lluvia en El Salvador (SNET, s.f).

\section{Resultados}

\section{Resultados de mediciones de tasa de evaporación}

Los resultados obtenidos confirman que la tasa de evaporación de agua bajo irradiación aumenta considerablemente cuando su superficie es recubierta con una película de grafito flotante. 


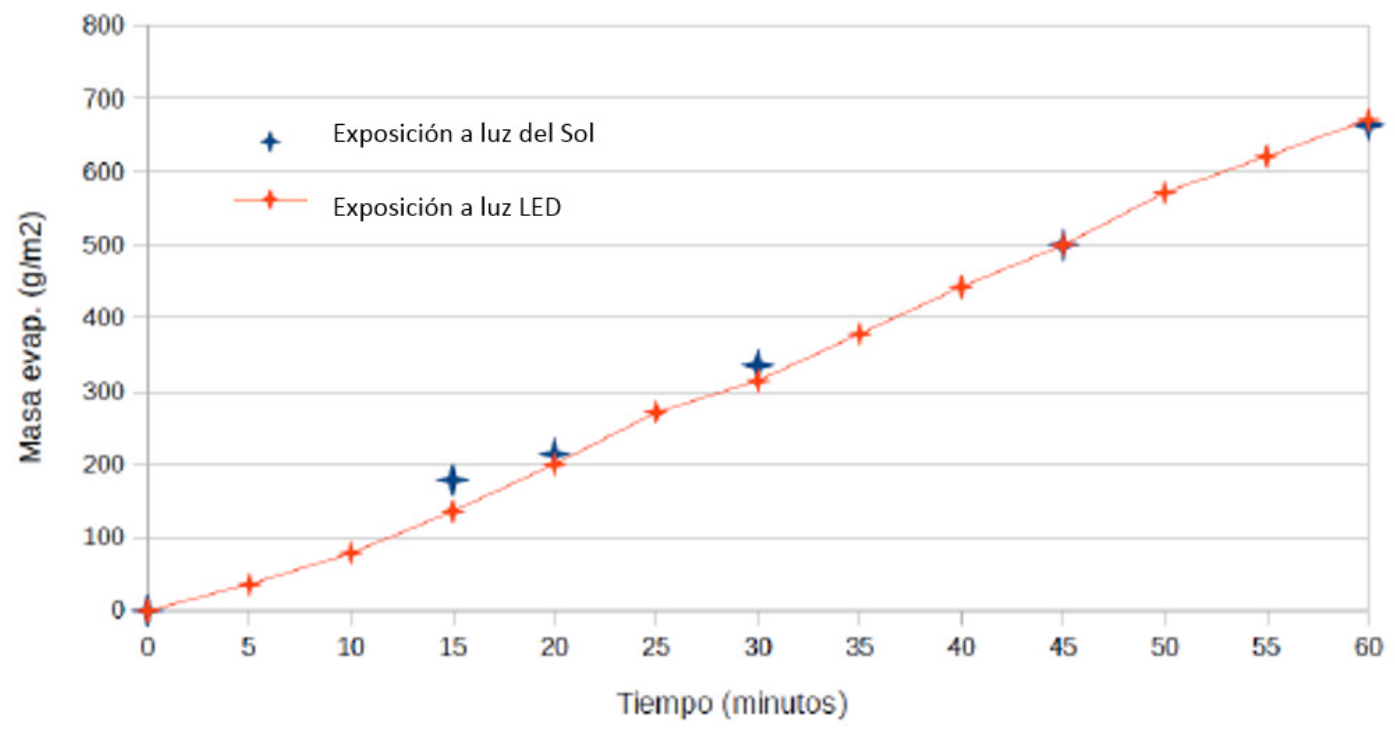

Figura n. $^{\circ}$ 3. Resultados normalizados de la evaporación de agua observada para el caso de exposición solar directa (puntos de medición marcados en color azul) y exposición a la luz proveniente de una lámpara LED de 100 Vatios posicionada a una distancia de $120 \mathrm{~mm}$ de la superficie de agua recubierta de grafito (puntos de medición en color rojo). En ambos casos se observa una evaporación por hora, inferior a 700 gramos de agua por metro cuadrado de superficie expuesta.

Sin la presencia de una película superficial de grafito, la tasa de evaporación de agua bajo irradiación es de aproximadamente 700 gramos de agua por metro cuadrado de superficie expuesta durante una hora (700 g/m2hora), tanto para el caso de exposición a la luz solar (9:00 - 10:00 am), como para el caso de exposición a la luz proveniente de la lámpara LED arriba descrita (ver Figura n. ${ }^{\circ}$ ).

Para el caso de superficies de agua recubiertas (ver Figura 4), la masa evaporada de agua, obtenida bajo condiciones experimentales idénticas alcanza valores de hasta $1314 \mathrm{~g} /$ m2hora, lo cual representa un aumento de hasta el $85 \%$ referente a los valores obtenidos con superficies de agua no recubiertas. En una primera aproximación se observa un comportamiento lineal entre la masa de agua evaporada durante el lapso de tiempo evaluado (60 minutos), así como diferencias significativas entre algunos de los materiales utilizados (ver Figura n. ${ }^{\circ}$ ).

\section{Resultados de observaciones estereomicroscópicas}

El estudio de las superficies mediante microscopía estereomicroscópica ${ }^{3}$ revela dos hechos importantes:

1. La remoción mecánica de las capas de grafito, inicialmente depositado en la superficie de agua, ocasiona que una fracción del material se hunda, formando un depósito de partículas en el fondo del contenedor, a una distancia de $3.2 \mathrm{~mm}$ de la superficie.

\footnotetext{
3 Estereomicroscopio trinocular marca Zeiss, Modelo Stemi 2000.
} 


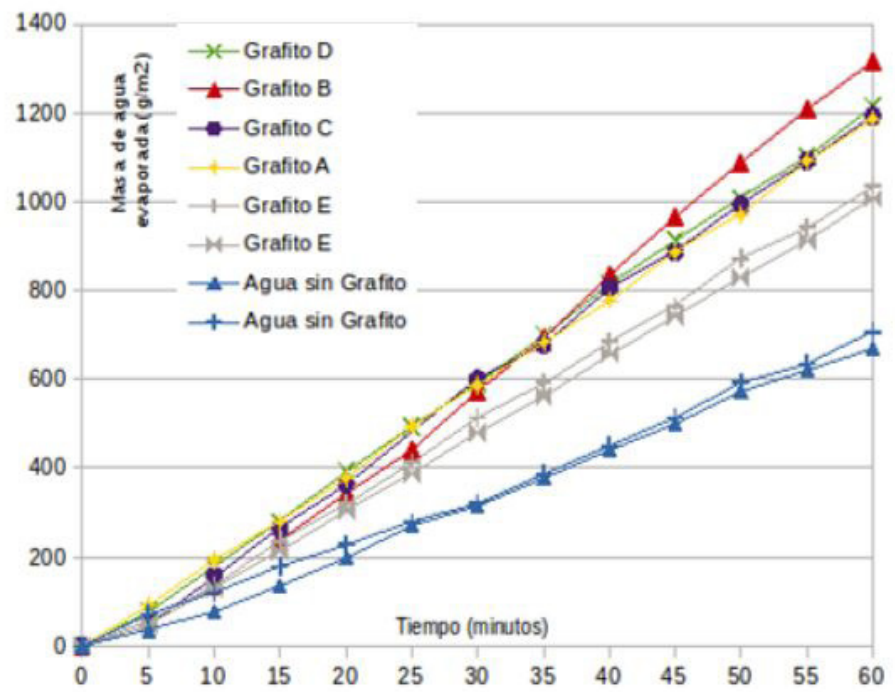

Figura n. ${ }^{\circ}$ 4. Comparación de las tasas de evaporación obtenidas con y sin películas de diferentes tipos de grafito. Se observa un comportamiento predominantemente lineal desde el inicio, así como diferencias en el comportamiento de las diferentes fuentes de materiales utilizados. El grafito proveniente de la fuente B (electrodo de batería) produce el mayor aumento de la tasa de evaporación de agua, mientras que el grafito proveniente de la fuente $\mathrm{E}$ (lubricante de cerraduras) ocasiona el menor efecto.

2. La observación microscópica de las películas flotantes revela que las partículas grafíticas se atraen fuertemente entre ellas, conformando una estructura porosa tridimensional (ver Figura n. ${ }^{\circ}$ ) que flota en la superficie, muy probablemente porque el peso específico de esta estructura es menor que la del agua. La estructura porosa de este recubrimiento podría resultar en un recubrimiento incompleto de la superficie de agua expuesta a la luz, y por ende una iluminación parcial de las partículas depositadas en el fondo del contenedor.

\section{Resultados de mediciones de temperatura}

Los resultados de mediciones de temperatura del agua del recipiente durante su irradiación (figura 6) muestran un aumento de la temperatura al inicio de unos $5^{\circ} \mathrm{C}$, seguido de un comportamiento estable durante el período de irradiación restante (55 minutos). Este comportamiento se atribuye a la presencia de un aislamiento térmico del volumen de agua ubicado por debajo de su superficie recubierta (ver Figura n. ${ }^{\circ}$ ).

\section{Discusión de resultados}

De acuerdo al estado actual de nuestro conocimiento, se ha logrado demostrar por primera vez que la presencia de películas flotantes hidrofóbicas de materiales grafíticos provenientes de fuentes comunes aumenta considerablemente la tasa de evaporación de agua bajo irradiación de luz.

Tomando en cuenta el amplio espectro de absorción de luz visible de los recubrimientos 


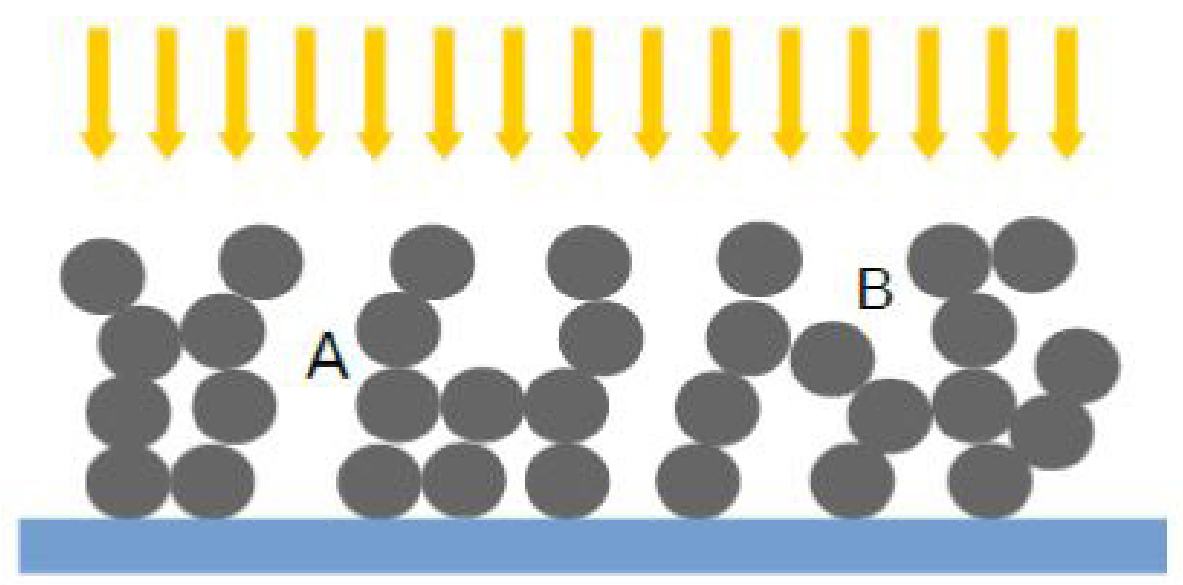

Figura n. $^{\circ}$ 5. Representación esquemática de la estructura tridimensional de las películas grafíticas flotantes, expuestas a irradiación (flechas amarillas). Las partículas individuales (círculos grises) se aglomeran en la superficie de agua (barra azul), creando una estructura tridimensional porosa. Se observa que algunas zonas de la superficie de agua no son recubiertas (Zona A) y expuestas a la irradiación. En otras zonas (B) se observa un recubrimiento completo de la superficie de agua.

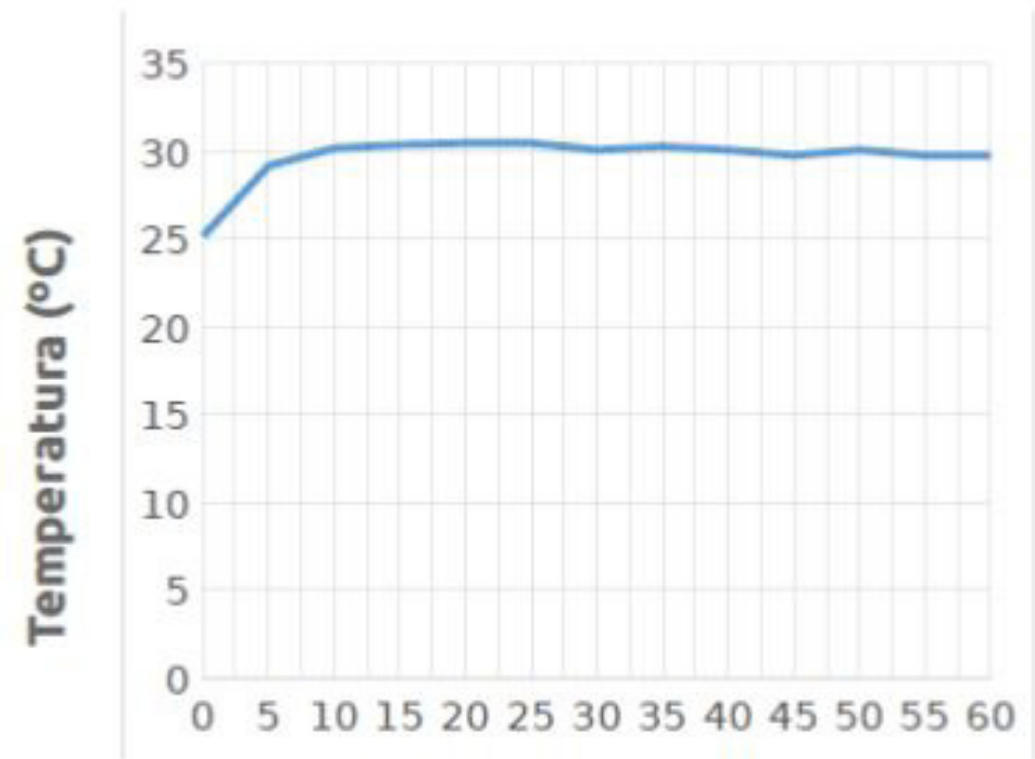

Figura . $^{\circ}$ 6. Desarrollo típico de la temperatura del agua debajo de la película durante la irradiación. Se denota un incremento de la temperatura durante los primeros 5 minutos, seguido por un comportamiento relativamente estable. 
(color negro), se atribuye el efecto observado a la generación de zonas calientes que facilitan la transición de la fase líquida del agua a la fase gaseosa, con menores pérdidas de energía por el calentamiento de las capas de agua subyacentes a la superficie. Esto explicaría parcialmente la diferencia de comportamiento entre los diferentes materiales utilizados: el material E (grafito lubricante), que causa el menor efecto, tiene una apariencia gris, debido a que está conformado por partículas planas, con superficies muy lisas, que reflejan parte de la luz incidente. En el caso del material B (proveniente de electrodo de batería) no se observa reflexión de luz.

La presencia de grafito, tanto en la superficie como en el interior de agua, podría dar lugar a diferentes mecanismos de evaporación de agua, que posiblemente se desarrollarían simultáneamente, así:

a) Mecanismo para las partículas sumergidas.

Asumiendo que entre las partículas sumergidas también figuran nanopartículas, se podría desarrollar un mecanismo propuesto recientemente por un grupo de investigadores de la universidad de Rice, USA (Neumann O. 2013): la iluminación de nanopartículas genera plasmones, elevando la temperatura de sus superficies muy arriba del punto de ebullición del agua. Esto ocasionaría la formación de una capa de vapor de agua alrededor de las partículas, prácticamente una burbuja, que actuaría un aislante térmico, reduciendo el calentamiento del agua líquida alrededor de ellas, y por ende, la pérdida de energía sin lograr evaporación. Al crecer con el tiempo, esta burbuja de vapor se fusiona con otras y se mueve hacia la superficie, emitiendo vapor.

b) Mecanismo para las partículas flotantes.

En el caso de las partículas flotantes, se podría desarrollar el mecanismo propuesto en el estudio realizado por el grupo de MIT (Ghasemi, 2014), que motivó el desarrollo de este estudio. La irradiación de las partículas de grafito sobre la superficie de agua genera sitios calientes (hot spots) donde la transición de la fase líquida a la fase gaseosa se desarrolla con mucha facilidad. La estructura porosa de la película actúa como aislante, previniendo la pérdida de energía por calentamiento del agua ubicado en la profundidad. E1 suministro constante de agua hacia la capa flotante se generaría por medio de fuerzas capilares a través de los poros de la película.

\section{Conclusiones}

Hemos presentado evidencia experimental para demostrar que la evaporación de agua bajo irradiación electromagnética, por ejemplo solar, puede ser aumentada considerablemente mediante la añadidura de materiales comúnmente disponibles en el país. Atribuimos este efecto a diferentes mecanismos que se basan en un aislamiento térmico de las superficies irradiadas. Además de promover la desalinización mediante irradiación solar, el efecto observado también podría utilizarse para fines de desinfección de superficies mediante el vapor generado, como lo proponen trabajos publicados recientemente por la Universidad de Rice, EE.UU.y otros (Neuman $A D$, 2013) (Anna Young, 2012) (Techonology Exchange Lab., s.f.). 
El entendimiento detallado de los resultados obtenidos debe incluir evidencia experimental sobre el mecanismo de transporte de agua a la superficie de la película grafítica. Resulta curioso medir la evaporación de cantidades relativamente grandes de agua (en el orden de 5*1013 moléculas/ $\mu \mathrm{m}^{2}$ hora) sin ocasionar cambios observables a nivel microscópico (aumento 100x) en la superficie de la película. Asumiendo que el área proyectada, promedio de las partículas que conforman la película grafítica, es de unos $100 \mu \mathrm{m} 2$ (10-10m2), esto implica que,en promedio, unas 1010 moléculas de agua son transportadas a lo largo de sus bordes en cada segundo, indicando la presencia de un mecanismo de transporte altamente eficiente.

Además de determinar la composición y la estructura submicroscópica de las películas grafíticas, por medio de métodos como SEM y AFM, queda por explorar la influencia de factores termodinámicos determinantes para incrementar la tasa de evaporación, como lo son la temperatura del agua y de las películas grafíticas, contenido de humedad del aire $y$ la influencia de un régimen de transporte de carácter convectivo, tanto para el agua como para el aire, a inmediaciones de la película.

En un siguiente paso se construirá un prototipo experimental que permita proceder con estudios, bajo condiciones de temperatura,presióny humedad de aire controladas. Las películas grafíticas también aparentan tener suficiente conductividad eléctrica como para realizar experimentos a futuro bajo condiciones de potencial controlado, y así poder estudiar las tasas de evaporación bajo condiciones oxidativas y reductivas en la interface entre el recubrimiento y el electrólito.

También se considera pedir el apoyo a otras organizaciones de investigación a niveles local y regional, para profundizar este tipo de estudio mediante el uso de otros materiales con propiedades definidas (ej. nanotubos de carbono), el estudio a nivel submicroscópico de las propiedades morfológicas de las películas superficiales y generar evidencia experimental sobre la posible generación de burbujas de vapor.

\section{Agradecimientos}

Se agradece al Instituto de Ciencia, Tecnología e Innovación de la Universidad Francisco Gavidia de E1 Salvador por el valioso apoyo brindado a esta iniciativa de investigación aplicada, así como a Lic. Mario R. Roldán por proporcionar las referencias 1,2 y 15.

\section{Referencias}

Anna Young. 2012. A solar powered autoclave to sterilize medical instruments in offgrid and rural clinics, Solarclave Nicaragua, fuente: https://d-lab. mit.edu/scale-ups/solarclave.

Aristoteles. 350 a.C. Ver "Meteorología" escrito por Aristoteles en el año 350 a.C, encontrado en: http:// classics.mit.edu/Aristotle/meteorology.2.ii.html

Cohen-Tanugi D. 2012. Water Desalination across Nanoporous Graphene, David CohenTanugi and Jeffrey C. Grossman, Nano Lett., 2012, 12(7), pp 3602-3608. DOI: 10.1021/ nn304948h·Fuente:PubMed

Cuéllar. 2001. La contaminación del agua en El Salvador: Desafios y respuestas institucionales. http://www.prisma.org.sv/uploads/media/bol43_ la_contaminacion_del_agua_en_ESV.pdf 
E.W. Webster, s.f. Material adicional de estudio publicado, referencia 3 .

El Salvador Mi País. S.f. Extensión territorial de El Salvador. http://www.elsalvadormipais.com/ extension-territorial-de-el-salvador

Ghasemi. 2014. Solar steam generation by heat localization. NATURE COMMUNICATIONS | 5:4449 | DOI: 10.1038/ncomms5449 | www. nature.com/naturecommunications. 2014.

Han D. 2011. Thermal properties of carbon black aqueous nanofluids for solar absorption, Dongxiao Han, Zhaoguo Meng, Daxiong Wu, Canying Zhang and Haitao Zhu, Nanoscale Research Letters 6(1):457·Julio 2011. DOI: 10.1186/1556-276X6-457 Fuente:PubMed

Neuman AD. 2013. Compact solar autoclave based on steam generation using broadband light-harvesting nanoparticles, Oara Neuman, Proceedings of the National Academy of Science of the United States of America (PNAS), vol. 110 no. 29, 11677-11681, doi: 10.1073/pnas.1310131110, mayo 2013.

Neumann O. 2013. Solar Vapor Generation Enabled by Nanoparticles, Oara Neumann et. al, American Chemical Society (ACS) Nano,
November 2012, DOI: 10.1021/nn304948h . Fuente: PubMed

Ruquan Ye. 2015. Coal as an abundant source of graphene quantum dots. James Tour et al. NATURE COMMUNICATIONS 4, Article number: 2943 doi:10.1038/ncomms3943

SILED. s.f. Especificaciones de lámpara LED: http://www.siled.com.mx/catalogos/potencia/files/ leds\%20de\%20potencia.pdf

SNET. s.f. Dato de humedad promedio del aire de servicio meteorológico de El Salvador. http://www. snet.gob.sv/meteorologia/climaelsal.htm

TAMEX. s.f. Especificaciones de balanza electrónica: http://www.tamex-precision.com.mx/ pdf/balanzas/Balanzas_de_Precision_Tamex.pdf

Techonology Exchange Lab. s.f .Solarclave Solar Autoclave: Innovations in International Health, Technlogy Exxchange Lab, Fuente: http:// www.techxlab.org/solutions/innovations-ininternational-health-solarclave-solar-autoclave 